\title{
Application Edge Designs to Study The Actual Causes That Led to The Frequent Traffic Accidents
}

\author{
Alanazi Talal Abdulrahman \\ Department of Mathamtics, College of Science, University of Ha'il, Saudi Arabia
}

\begin{abstract}
Cite This Paper in the following Citation Styles
(a): [1] Alanazi Talal Abdulrahman, "Application Edge Designs to Study The Actual Causes That Led to The Frequent Traffic Accidents," Civil Engineering and Architecture, Vol.9, No.4, pp. 1057-1063, 2021. DOI: 10.13189/cea.2021.090408

(b): Alanazi Talal Abdulrahman, (2021). Application Edge Designs to Study The Actual Causes That Led to The Frequent Traffic Accidents. Civil Engineering and Architecture, 9(4), 1057-1063. DOI: 10.13189/cea.2021.090408
\end{abstract}

Copyright $@ 2021$ by authors, all rights reserved. Authors agree that this article remains permanently open access under the terms of the Creative Commons Attribution License 4.0 International License

\begin{abstract}
Background: Hundreds of people die every day as a result of traffic collisions all over the country. The majority of these collisions are caused by cars or pedestrians breaking traffic laws, such as speeding or talking on their phones.. Most drivers drive their cars while feeling sleepy or tired, or driving under the influence of medication that causes drowsiness, or lack of concentration, or hallucinations. The latest statistics from Ministry of health of Kingdom of Saudi Arabia indicate that the death rate in road accidents in Saudi Arabia is 17 people every 40 minutes. Therefore, based on this statistic, traffic accidents have become a source of concern for the Saudi Arabian society. They cause great losses in human and material resources, in addition to psychological and social problems. It is necessary to conduct a field study to find out the actual cause of the spread of accidents in Saudi Arabia. Objective: This study sets out to examine the actual reasons that led to the frequent traffic accidents in the Saudi Arabian society. Methods: An online survey provided quantitative data from 1000 participants. Factual importance was investigated utilizing edge plan examination and the relapse investigation utilizing the SPSS program to decide the real makes that drove the spread of auto collisions in the Saudi Arabian culture. Results: The findings indicated that driving vehicles by unqualified persons, drug abuse and grain deprivation are the factors that led to the spread of traffic accidents in the Saudi Arabian society. Conclusion: it is recommended that Saudi Arabian government professionals develop an operational plan to study these causes to take action. In the future, data may be analyzed using supersaturated prototypes, in which a large number of variables are studied for just a few simulation trials. Keywords Traffic Accidents, Edge Designs, Linear Model, Screening Design, Data Analysis
\end{abstract}

\section{Introduction}

Traffic accidents are a major cause of death and disability among the population of most nations across the globe. Most of the crashes experienced on roads are usually fatal and result in death or injury for both drivers and pedestrians. According to[1], Saudi Arabia has seen a significant rise in road deaths, which has reached an unprecedented number. As a result, the authorities have made efforts to implement policies that will make it compulsory for people to report every accident and seek medical assistance from government healthcare institutions. The data collected from the police and the medical institutions could then be used to evaluate the causes of the accidents and assist the government in adopting the right measures to reduce or eliminate such incidences ([2]). By successfully reducing the rate of accidents in the country, the government can significantly lower the amount of resources used to treat victims of such misfortunes. Furthermore, the decline in accident cases can help lower the psychological and social stress that is suffered by the families of the victims.

Saudi Arabia's economy has recorded tremendous growth over the last three decades due to the income it realized from the sale of oil. This has led to an increase in the individual incomes and, subsequently, the standard of living of the country's citizens. Additionally, the population of the country has increased over time, and the number of vehicles on the roads has significantly risen. According to [3], there has been a rapid expansion of the existing road infrastructure to facilitate the smooth movement of people and goods. However, this rapid 
increase in the number of cars on Saudi roads has led to a rise in accident cases. The majority of accidents reported involved vehicle collisions, cars running over pedestrians, vehicles hitting walls and electric posts, and animals being run over ([13]). To address this problem, the government made it mandatory for witnesses to report accidents, whether minor or major, to the relevant authorities. [6] note that the data collected from the aforementioned reports made it possible for the ministry of transport to create an electronic database. The information contained therein could be used to help the government to make appropriate policies to prevent accidents from occurring in the future.

\begin{tabular}{|c|c|c|c|c|c|}
\hline \multirow{2}{*}{ Region } & \multirow[b]{2}{*}{ Total } & \multicolumn{2}{|c|}{ Outside the City } & \multicolumn{2}{|c|}{ Inside the City } \\
\hline & & p.c & $\begin{array}{c}\text { No. Of } \\
\text { Accidents }\end{array}$ & p.c & $\begin{array}{l}\text { No. of } \\
\text { Accidents }\end{array}$ \\
\hline Riyadh & 147568 & $8.00 \%$ & 8594 & $33.78 \%$ & 138974 \\
\hline Makkah & 126537 & $22.28 \%$ & 23930 & $24.94 \%$ & 102607 \\
\hline Madinah & 19058 & $6.44 \%$ & 6921 & $2.95 \%$ & 12137 \\
\hline Al-Qasim & 24273 & $9.29 \%$ & 9978 & $3.47 \%$ & 14295 \\
\hline Eastern & 88065 & $18.42 \%$ & 19785 & $16.60 \%$ & 68280 \\
\hline Aseer & 32163 & $12.44 \%$ & 13357 & $4.57 \%$ & 18806 \\
\hline Tabouk & 20638 & $3.46 \%$ & 3714 & $4.11 \%$ & 16924 \\
\hline Hael & 8415 & $2.83 \%$ & 3037 & $1.31 \%$ & 5378 \\
\hline Northern Boarders & 13076 & $1.69 \%$ & 1816 & $2.74 \%$ & 11260 \\
\hline Jazan & 22229 & $11.67 \%$ & 12529 & $2.36 \%$ & 9700 \\
\hline Najran & 3220 & $1.15 \%$ & 1231 & $0.48 \%$ & 1989 \\
\hline Al-Baaha & 4166 & $1.01 \%$ & 1087 & $0.75 \%$ & 3079 \\
\hline Al-Jowf & 9387 & $1.32 \%$ & 1419 & $1.94 \%$ & 7968 \\
\hline Total & 518795 & $100 \%$ & 107398 & $100 \%$ & 411397 \\
\hline
\end{tabular}

Figure 1. Incidences of injury and death reported all cities of Saudi Arabia

The high accident figures in Saudi Arabia have raised concerns among all members of the country. Incidences of injury and death are reported across all cities of the nation (See Figure 1 ). Thus, it is necessary to conduct a field study to determine the main causes of the problems. The investigation should be conducted while utilizing quantitative data to determine the reasons behind the rise in recorded cases of traffic accidents. The paper begins by reviewing previous research on the causes of accidents in the country.

Visitors to Saudi Arabia are also taken aback by the country's high number of accident incidents, as well as their severity in terms of death and serious injuries. These mishaps are so prevalent in the country's major cities that barely a day goes by without an incident being reported ([15]). As a result, visitors have suggested that road accidents should be declared a national disaster in the country. According to the data published by the Saudi Arabia Ministry of health, the death rate due to road accidents in the country is 17 people per 40 minutes ([1]). Furthermore, [1] state that over 68000 people suffer accidentrelated injuries in Saudi Arabia annually; thus, the country has the highest number of road accident causalities across the globe. The nation's government healthcare systems are under considerable pressure as a result of the high casualty rate.

The victims of road accidents have risen in Saudi Arabia over the past two decades. According to [12], the number of car accident-related deaths in Saudi Arabia-such as vehicle collisions, cars hitting a wall or post, and pedestrians being run over by a speeding car currently stands at 86000 . Similarly, states that a total number of 611000 people got injuries from accidents in the country, with $7 \%$ of these injuries resulting in permanent disability. The above figure is alarming, especially since they exceed the number of deaths recorded in the Argentinian, Western Saharan, India-Pakistan, and Gulf wars, as well as the Nepalese Civil war and the independence war of Croatia. [17] proposes that the high rate of deaths and material losses, which have been estimated at 13 billion riyals a year, from car accidents in Saudi Arabia, makes it imperative for the relevant national authorities to adopt measures to curb this problem.

Human causes are the most important contributors to road accidents, which are the leading cause of death for Saudi Arabia's young people. A scientific study has established that accidents have increased in recent times due to the negligence of traffic rules by drivers and pedestrians. The drivers contribute to accidents by driving under the influence of medication and while suffering from visual challenges ([10]). Additionally, drivers cause accidents when they get distracted by conversations with their passengers while driving, chirp in a chaotic way, make abrupt stops in dangerous areas, and use their mobile phones. [9] study on the Dhi Qar province also revealed that accidents could occur due to lack of periodic inspection or modification of cars their original state. On the other hand, a reckless pedestrian could contribute to an accident by crossing roads in non-designated places. The failures to identify and identify the factors that contribute to traffic accidents have increased the number of deaths and injuries in Saudi Arabia. [11], in his article "Car accident in the city of Riyadh, causes effects, and solutions," suggests that accidents could be reduced by identifying their causal factors and listing them in the order of the extent of their influence. The compiled list could help the government in formulating policies and ensuring their implementation to drastically minimize the accident cases in the country. For example, the result of the study on the causes and extent of accidents in the city of Riyadh suggests that it needs a pause 50 to minimize their occurrence ([2]). Furthermore, the Saudi Arabia government should enact a national strategy with goals to address traffic accidents. The strategy should also be implemented in a planned schedule to enhance its effectiveness. Failure to train prospective drivers adequately and sufficiently before granting them a driver's license has contributed significantly to an increase in the number of road accidents. According to the[7] article, which is titled " Car accidents in the Islamic Criminal jurisprudence Comparative', 'the adequate training of drivers increases their competence and makes them more wary of traffic regulations. This training should be conducted in an approved traffic school and aimed at enhancing practical and theoretical knowledge. Drivers who have accurate information on- road use and vehicle maneuvering characteristics are likely to adhere to traffic rules, thus decreasing instances of accidents. [18] assert that competent drivers can identify a potential cause of an accident and are put the necessary corrective measures to avoid those situations. The lack of understanding of people's feelings contributes to the occurrence of traffic accidents. [9], in his article titled "Traffic Accident in the City of Benghazi, its Causes, Treatment and Prevention," accidents are caused by the social, economic, and psychological phenomenon. The driver's emotions affect his 
or her ability to steer the vehicle. The high rates of car crash in the region suggest that most drivers have a cynical feeling that negatively affects their driving ability. The communities need to make effort to ensure that drivers have positive emotions, which is likely to increase their level of concentration while driving. People who drive when emotionally stable are significantly less likely to be involved in accidents than those who do not. A driving culture that encourages overspeeding on roads contributes to the occurrence of a traffic accident. A study conducted in Saudi Arabia's Jeddah region presents a positive correlation between cultures that encourages over speeding and high rates of accidents. According to [2] the urban lifestyle in towns and cities encourages overspeeding while the culture outside the cities encourages motorists to drive at an average speed. Statistics from the research by [4] show that $80 \%$ of fatal accidents occur in major towns due to the unwillingness of drivers to obey traffic regulations. Conversely, only $18 \%$ of accidents occur outside the cities. The evaluation consequences of [14] study confirmed that the excessive severity of visitors injuries in Ambon City turned into 91.95\% utilized by motive force conduct elements. Three different elements that purpose visitors injuries encompass drunkenness, carelessness, and drowsiness. The high road accident figures obtained from the aforementioned studies show that Saudi Arabia is the leading country in the world with regard to road accident deaths. [16] study confirmed that the World Health Organization (WHO) detailed that 1.24 million individuals were executed out and about, and up to 50 million individuals were harmed around the world, and the quantity of street traffic passings is required to increment further by 2020 . Almost $3 / 4$ of in general street passings happen in agricultural nations, despite the fact that street passings are basic in created nations. Road traffic setback in the Kingdom of Saudi Arabia (KSA) addresses $4.7 \%$, taking everything into account, while road traffic fatalities don't outperform $1.7 \%$ in Australia. Saudi Arabia was found to have higher number of passings from RTAs among significant group compensation states (incident to death extent is 32:1 versus 283:1 in USA), and is seen as the country's essential driver of death for 16-30-year-old folks.

\section{Kinds of traffic accidents}

1 - Collisions between vehicles: these incidents occur as a result of the collision of two vehicles, or one collides with the other, and this is the most frequent incidents OCCUT.

2 -Run over: this is a road accident that happens when a vehicle collides with another vehicle while driving on the highway, either deliberately or accidentally.

3- Collision with something: this type is the result of a car collision with something fixed on the street, such as electricity or a wall.

4 - Animal collisions: the result of a vehicle colliding with a passing animal on the street.

\section{Method}

\section{Target population and study sample}

A survey was utilized in this examination on the grounds that the reseacher pointed requested that members decide the spread of the wonder of traffic accidents. The survey consisted of two parts that ask participate ( male and female driver license ) to completet questions :the value of buying your car and his/her opinion of the most important reasons that led to the large number of traffic accidents according to 12 reasons. The target masses was 1000 individuals on 9/2020 in Ha'il.

\section{Data collection}

The questionnaire is sent to the participants and then the questions are answered based on their opinion by answering yes to the actual reason or no to the inactive reason. This is shown in the table 1, which shows the information gathered.

\section{How to select a sample}

A pre-design consisting of four factors and eight trials is selected. This design is considered in all the data that has been put in the previous steps and in case of obtaining the whole design is placed the value of the response and the number of the person who answered the questionnaire.

Consolidating and adjusting distinctive examination technique

The design chosen in the previous step is analyzed using the edges dsigns and regression methods. If the factors that we get from the edge design method are the same as the regression method, these factors are the actual causes of traffic accidents in the Saudi society.

\section{Results}

The researcher selects the edges design that contains four factors and 8 runs and then searches for it in the completed questionnaire. The select plan was dissected utilizing edge plan investigation and the relapse examination utilizing the SPSS program to decide the genuine makes that drove the spread of auto collisions in the Saudi society. [8] have introduced the edge plans. The edge depends upon a model-independent test that can be used for dynamic components. To know the powerful factors, the assessments are coordinates into a social event of $E$ sets. In this procedure the assessments contrasts in a solitary part. It is ordinary in screening tests, and recommends that for all intents and purposes all differentiations

$$
z_{r, c}:=y_{r}-y_{c},(r, c) \in E,
$$

involve uproar figuratively speaking. If the scientist acknowledge that the disturbance in the data is added substance, commonly dispersed with zero mean and contrast variances (Var) the $n-p$ of the $z_{r, c}$ are consistently appropriated with zero mean and vacillation $2 \sigma^{2}$. Because of the dark number of exemptions, the change ought to be evaluated in an incredible way. For example, the specialist can use the median estimate(ME)

$$
\widetilde{M E}=\frac{\operatorname{med}\left\{\left|z_{r, c}\right|:(r, c) \in E\right\}}{2^{\frac{1}{2}} \times 0.675} .
$$

More insights regarding this strategy can be found in [5]. In order to address these ethical concerns, the following example are taken. 


\begin{tabular}{|l|l|l|}
\hline Factor & signal & Description \\
\hline $\begin{array}{l}\text { Absence of physical deterrent in traffic ac- } \\
\text { cidents }\end{array}$ & + & Yes \\
& - & No \\
\hline The driver feels tired and tired & + & Yes \\
& - & No \\
\hline Mobile usage & + & Yes \\
& - & No \\
\hline Excessive speed and recklessness & + & Yes \\
& - & No \\
\hline Infringement of irrigated laws & + & Yes \\
& - & No \\
\hline $\begin{array}{l}\text { The road where the vehicles are traveling } \\
\text { for many of the things that lead to accident }\end{array}$ & + & Yes \\
accidents and maintenance of the road lack & & \\
of peace factors on the road & - & \\
\hline Weather & + & No \\
\hline Driving vehicles by unqualified persons & + & Yes \\
\hline Drug abuse and grain deprivation & - & No \\
\hline Vehicle leadership by the elderly and the & + & Yes \\
visually impaired & - & No \\
\hline The presence of animals in the roadt & - & Yes \\
\hline Criminal pursuits by police and spoilers & + & No \\
\hline Y(Response) & - & Yes \\
\hline & Number & No \\
\hline
\end{tabular}

Table 1. The level of the causes for traffic accidents

Example 1 : Let $n=4$ and weather $x_{1}$, driving vehicles by unqualified persons $x_{2}$, drug abuse and grain deprivation $x_{3}$, vehicle leadership by the elderly and the visually impaired $x_{4}$. Dissecting the information in Table 2 utlizing edge design is

\begin{tabular}{|c|c|c|c|c|c|c|}
\hline Run & $x_{1}$ & $x_{2}$ & $x_{3}$ & $x_{4}$ & $\mathrm{Y}$ & Reaction number \\
\hline 1 & + & + & + & + & 66000 & 11 \\
2 & - & + & + & - & 60000 & 75 \\
3 & - & - & + & + & 75000 & 818 \\
4 & - & + & - & + & 77000 & 58 \\
5 & - & + & + & + & 70000 & 24 \\
6 & - & - & + & - & 80000 & 108 \\
7 & - & - & - & + & 90000 & 155 \\
8 & - & + & - & - & 80000 & 36 \\
\hline
\end{tabular}

Table 2. One recreate for Example 1.

the accompanying. Firstly, the auther finds every one of the six differences of the reaction $y$ over the edges and the total esteem, as gave in Table 3 . Secondly, the author figures the middle to expect the number $p$ as dynamic components. Thirdly, the auther find $\mathrm{ME}$ and ascertain $G(p)$ and $k * 2^{0.5} \sigma$. At last, if the $G(p)$ is more noteworthy than $\mathrm{p}$ for some theory $\mathrm{p}$, the author stops the technique and find the dynamic factor. Table 4 demonstrates the outcomes; the auther has $\mathrm{G}(3)=2$, which implies there are active factors: driving vehicles by unqualified persons and drug abuse and grain deprivation.

\begin{tabular}{|c|c|c|c|}
\hline$x_{1}$ & $x_{2}$ & $x_{3}$ & $x_{4}$ \\
\hline-4000 & -20000 & -15000 & -3000 \\
\hline 4000 & 20000 & 15000 & 3000 \\
\hline
\end{tabular}

Table 3. Model-self-sufficient checks with the edge plan in Table 1

\begin{tabular}{|c|c|c|c|c|c|}
\hline$p$ & Median & $\widetilde{M} E(p)$ & $k 2^{\frac{1}{2}} \widetilde{M} E(p)$ & $G(p)$ & $G(p)<p ?$ \\
\hline 0 & 9500 & 9951.87 & 14074.07 & 2 & No \\
1 & 4000 & 4190.26 & 5925.92 & 2 & No \\
2 & 35500 & 3666.47 & 5185.18 & 2 & No \\
3 & 3000 & 3142.69 & 4444.44 & 2 & Yes \\
\hline
\end{tabular}

Table 4. Little by little calculations for the examination with the edge of the arrangement

Presently an investigation information in Table 2 (utilizing liner regression) with the product bundle SPSS that uncovered there are dynamic factors driving vehicles by unfit peo- 
ple and medication misuse and grain hardship and give an expected liner model $Y=78000-6000 x_{2}-6500 x_{3}+\epsilon$, with $R-s g=89 \% \epsilon$ of mean 74750 and standard deviation $\sigma=9331.20$.

Additionally from the outcome, the lingering is ordinary since p-value (0.200) is more than 0.05 .

From the abovementioned, the author sees that the strong edge plan strategy shows dynamic elements: driving vehicles by unqualified persons and drug abuse and grain deprivation and the regression technique shows no dynamic factors driving vehicles by unqualified persons and drug abuse and grain deprivation. So the author concludes that there are dynamic elements driving vehicles by unqualified persons and drug abuse and grain deprivation with linear contributions.

\section{Example 2:}

Let $n=4$ and driving vehicles by unqualified persons $x_{1}$ , drug abuse and grain deprivation $x_{2}$, vehicle leadership by the elderly and the visually impaired $x_{3}$, the presence of animals in the road $x_{4}$. Dissecting the information in Table 5 as

\begin{tabular}{|c|c|c|c|c|c|c|}
\hline Run & $x_{1}$ & $x_{2}$ & $x_{3}$ & $x_{4}$ & $\mathrm{Y}$ & Response number \\
\hline 1 & + & + & + & + & 126000 & 10 \\
2 & - & + & + & - & 70000 & 459 \\
3 & - & - & + & + & 80000 & 558 \\
4 & - & + & - & + & 90000 & 88 \\
5 & - & + & + & + & 60000 & 2 \\
6 & - & - & + & - & 120000 & 370 \\
7 & - & - & - & + & 100000 & 120 \\
8 & - & + & - & - & 100000 & 196 \\
\hline
\end{tabular}

Table 5. One recreate for Example 2.

been described previously. the results are shown in tables 6 and 7 . The author has $\mathrm{G}(3)=2$ active factors:driving vehicles by unqualified persons and drug abuse and grain deprivation. Presently an examination information in Table 5 (utilizing liner regression) with the product bundle SPSS that uncovered there is dynamic variable driving vehicles by inadequate people and give an expected liner model $Y=128000+34250 x_{1}+\epsilon$, with $R-s g=87.9 \% \epsilon$ of mean 93250 and standard deviation $\sigma=23026.38$.

Additionally from the outcome, the leftover is typical in light of the fact that p-value (0.200) is more than 0.05 .

From the abovementioned, the author sees that the strong edge plan strategy shows dynamic elements driving vehicles by unqualified persons and drug abuse and grain deprivation and the regression technique shows dynamic factor driving vehicles by unqualified persons. The author concludes that there is dynamic element driving vehicles by unqualified persons with a linear contribution.

\begin{tabular}{|c|c|c|c|}
\hline$x_{1}$ & $x_{2}$ & $x_{3}$ & $x_{4}$ \\
\hline 66000 & -50000 & -20000 & -10000 \\
\hline 66000 & 50000 & 20000 & 10000 \\
\hline
\end{tabular}

Table 6. Model-self-sufficient checks with the edge plan in Table 5

\begin{tabular}{|c|c|c|c|c|c|}
\hline$p$ & Median & $\widetilde{M} E(p)$ & $k 2^{\frac{1}{2}} \widetilde{M} E(p)$ & $G(p)$ & $G(p)<p ?$ \\
\hline 0 & 35000 & 36664.79 & 103703.70 & 0 & No \\
1 & 20000 & 20951.31 & 59259.25 & 1 & No \\
2 & 15000 & 15713.48 & 44444.44 & 2 & No \\
3 & 10000 & 10475.65 & 29629.62 & 2 & Yes \\
\hline
\end{tabular}

Table 7. Little by little calculations for the examination with the edge of the arrangement

\section{Example 3 :}

Let $n=4$ and drug abuse and grain deprivation $x_{1}$, vehicle leadership by the elderly and the visually impaired $x_{2}$, the presence of animals in the road $x_{3}$, criminal pursuits by police and spoilers $x_{4}$. Dissecting the information in Table 8 as

\begin{tabular}{|c|c|c|c|c|c|c|}
\hline Run & $x_{1}$ & $x_{2}$ & $x_{3}$ & $x_{4}$ & Y & Response number \\
\hline 1 & + & + & + & + & 15000 & 144 \\
2 & - & + & + & - & 72000 & 266 \\
3 & - & - & + & + & 68000 & 240 \\
4 & - & + & - & + & 76000 & 638 \\
5 & - & + & + & + & 170000 & 246 \\
6 & - & - & + & - & 40000 & 53 \\
7 & - & - & - & + & 40000 & 596 \\
8 & - & + & - & - & 120000 & 754 \\
\hline
\end{tabular}

Table 8. One recreate for Example 3.

been described previously. the results are shown in tables 9 and 10. The author has $G(3)=2$ active factors: driving vehicles by drug abuse and grain deprivation and criminal pursuits by police and spoilers. Presently an examination information in Table 8 (utilizing liner regression) with the product bundle SPSS that uncovered there is no dynamic variable and give an expected liner model $Y=15000+\epsilon$, with $R-s g=70.6 \% \epsilon$ of mean 75125 and standard deviation $\sigma=49510.28$.

Additionally from the outcome, the lingering is typical on the grounds that p-value $(0.200)$ is more than 0.05 .

From the abovementioned, the author sees that the strong edge plan strategy shows dynamic elements drug abuse and grain deprivation and criminal pursuits by police and spoilers and the regression technique shows no dynamic factor. The author concludes that there is no dynamic element with a linear contribution.

\begin{tabular}{|c|c|c|c|}
\hline$x_{1}$ & $x_{2}$ & $x_{3}$ & $x_{4}$ \\
\hline-155000 & 32000 & 28000 & -44000 \\
\hline 155000 & 32000 & 28000 & 44000 \\
\hline
\end{tabular}

Table 9. Model-self-sufficient checks with the edge plan in Table 5 


\begin{tabular}{|c|c|c|c|c|c|}
\hline$p$ & Median & $\widetilde{M} E(p)$ & $k 2^{\frac{1}{2}} \widetilde{M} E(p)$ & $G(p)$ & $G(p)<p ?$ \\
\hline 0 & 38000 & 39807.49 & 56296.29 & 1 & No \\
1 & 32000 & 33522.09 & 47407.40 & 1 & No \\
2 & 30000 & 31426.96 & 44444.44 & 1 & No \\
3 & 28000 & 29331.83 & 41481.48 & 2 & Yes \\
\hline
\end{tabular}

Table 10. Little by little calculations for the examination with the edge of the arrangement

\section{Discussion}

ccording to the strong road injury figures collected from the above reports, Saudi Arabia is the world's leading country in terms of road accident deaths. During the investigation, the main objective was to identify the main factors that contributed to the increase in road accidents over the last few decades in Saudi Arabia. The research has identified human factors such as driving under the influence of medication and negligence, characterized by a failure to take the vehicle for inspection, as the chief human factors that contribute to road accidents. Additionally, failure to identify and rank the factors that lead to accidents based on their level of influence has contributed to an increase in the cases of accidents in the country. Furthermore, accidents have risen in the country due to a failure to train drivers adequately and sufficiently. Moreover, drivers' emotions could affect their ability to drive. Lastly, a culture that encourages over speeding has been noted to contribute to higher cases of traffic accidents. Understanding the causes of the increased rate of accidents in Saudi Arabia could help the relevant authorities adopt suitable measures to reduce the death and injuries arising from vehicle accidents in the future. On this basis, it is recommended that Saudi Arabia government develop an operational plan to study these causes. In future investigations, it might be possible for data to use supersaturated designs where numerous components are researched utilizing a couple of exploratory runs. Furthermore, there is a requirement for more research thinks about that addresses different parts of diabetes in the Saudi setting, for example, the extent of this phenomenon with three levels $(+, 0,-)$.

\subsection{Acknowledgment}

I might want to thank the University of Ha'il in Saudi Arabia for monetarily supporting my exploration project BA-1903.

\subsection{Ethics endorsement and agree to participate}

Not appropriate

\subsection{Availability of information and material}

The information upholds the finding of this examination are accessible inside the article and its beneficial material.

\subsection{Competing interests}

Not relevant

\subsection{Funding}

Not relevant

\section{REFERENCES}

[1] Ageli, M. and Zaidan, A. (2013). Road traffic accidents in saudi arabia: An ardl approach and multivariate granger causality. Ageli, Mohammed. Moosa (2013).'Road Traffic Accidents in Saudi Arabia: An ARDL Approach and Multivariate Granger Causality'. Journal of Economics and Finance, 5(7).

[2] Al Ghamdi, M. and Al Ghamdi, S. (2014). Traffic culture and its relationship to traffic accidents in jeddah. pages 413.

[3] Al Turki, Y. A. (2014). How can saudi arabia use the decade of action for road safety to catalyse road traffic injury prevention policy and interventions? International journal of injury control and safety promotion, 21(4):397-402.

[4] Al-Zahrani, A., Jamjoom, M., and Al-Bar, H. (1994). Traffic accident characteristics in jeddah, saudi arabia. Engineering Sciences, 6(1).

[5] Alanazi, T., Georgiou, S., and Stylianou, S. (2018). Construction and analysis of edge designs from skewsymmetric supplementary difference sets. Communications in Statistics-Theory and Methods, 47(20):5064-5076.

[6] Ansari, S., Akhdar, F., Mandoorah, M., and Moutaery, K. (2000). Causes and effects of road traffic accidents in saudi arabia. Public health, 114(1):37-39.

[7] Asseedah, N. M. H. (2009). Car accidents in the islamic criminal jurisprudence comparative turispradence study.

[8] Elster, C. and Neumaier, A. (1995). Screening by conference designs. Biometrika, 82(3):589-602.

[9] Faraj, S. (2015). Traffic accidents in the city of benghazi, its causes and treatment and prevention.

[10] Hassan, M. A. M. A.-H. (2010). Breach in the holy quran.

[11] Imad, A. (2015). Car accidents in the city of riyadh causes, effects and solutions. 10(3):2-13.

[12] Issa, Y. (2016). Effect of driver's personal characteristics on traffic accidents in tabuk city in saudi arabia. Journal of transport literature, 10(3):25-29.

[13] Khawag, W. Y. (2017). The problem of traffic congestion in saudi arabia. International Journal of Scientific, Engineering Research, 8(9):1632-1637. 
[14] Lenora Leuhery, H. (2020). Determination of black site area based on equivalent accident number analysis: Case study national roads in ambon city. Civil Engineering and Architecture, 8(5):1063-1073, DOI: 10.13189/cea.2020.080533.

[15] Mansuri, F. A., Al-Zalabani, A. H., Zalat, M. M., and Qabshawi, R. I. (2015a). Road safety and road traffic accidents in saudi arabia: a systematic review of existing evidence. Saudi medical journal, 36(4):418.

[16] Mansuri, F. A., Al-Zalabani, A. H., Zalat, M. M., and
Qabshawi, R. I. (2015b). Road safety and road traffic accidents in saudi arabia: a systematic review of existing evidence. Saudi medical journal, 36(4):418.

[17] Ofosu, J., Abouammoh, A., and Bener, A. (1988). A study of road traffic accidents in saudi arabia. Accident Analysis \& Prevention, 20(2):95-101.

[18] Rubin, H. J. and Rubin, I. S. (2011). Qualitative interviewing: The art of hearing data. Sage. 